\title{
Compact Bioimplantable MICS and ISM Band Antenna Design for Wireless Biotelemetry Applications
}

\author{
Merih PALANDOKEN \\ Dept. of Electrical and Electronics Engineering, University of Izmir Katip Celebi, 35620, Izmir, Turkey \\ merih.palandoken@ikc.edu.tr \\ Submitted September 19, 2017 / Accepted September 19, 2017
}

\begin{abstract}
A compact dual-band bioimplantable antenna with novel resonator geometry is designed for dual-band wireless biotelemetry applications in MICS and ISM bands. The radiating element geometry is based on an Lshaped transmission line fed anti-spiral resonator structure, which is loaded with the spiral resonator at the end to increase the effective electrical length. The effect of resonator geometric parameters on the return loss is discussed with the inclusion of three layered lossy human model in the numerical calculations. The footprint size of the optimized bioimplantable antenna is $15 \times 15 \times 1.92 \mathrm{~mm}^{3}$, having the total surface area of $\lambda_{0} / 20 \times \lambda_{0} / 20$, where $\lambda_{0}$ is the free space wavelength at $403 \mathrm{MHz}$ in MICS band. The compact dual-band antenna has the impedance bandwidth of $24.81 \%$ at $403 \mathrm{MHz}$ and $14.7 \%$ at $2.45 \mathrm{GHz}$ with the gain values $-12.25 \mathrm{dBi}$ and $-12.4 \mathrm{dBi}$ in MICS and ISM bands, respectively. The average $S A R$ values at the resonance frequencies are numerically computed to find out the input power delivered to the antenna for the reliable operation. The radiation parameters and $3 D$ radiation patterns indicate the potential use of the proposed implantable antenna with the permissible gain in dual-band wireless biotelemetry applications.
\end{abstract}

\section{Keywords}

Dual-band bioimplantable antennas, MICS and ISM bands, human body model, electrically small resonator

\section{Introduction}

During the last decades, the ever-evolving miniaturization of wireless portable electronic devices with increasing multiple functionalities has received great attention in the wide variety of different technological applications. One important application field of wireless communication technologies is the biotelemetry application.

In the biomedical domain, the bioimplanted device located inside the human body by the surgical operations has the main functionality of performing the various diagnostic and therapeutic functions to transmit the biological and physiological signals robustly to the exterior monitoring and controlling unit in the health care system [1]. Therefore, the miniaturized electrically small antenna is the key component of medical implant device (MID) in the wireless telemetry applications. While the main concern of biotelemetry application is the human health care, the implantable antennas have to be specifically designed satisfying the various physical and biological constraints inside the human body in addition to RF electrical regulations of FCC (Federal Communications Commission) for the implanted devices [2]. The ITU-R Recommendation SA.1346 [3] has outlined the pre-allocated frequency band of (402 to 405) $\mathrm{MHz}$ frequency band for Medical Implant Communication Service (MICS), regulated by FCC [4] and ERC (European Radio-Communication Commission) [5]. The frequency bands of (433.1-434.8) MHz, (868-868.6) MHz and (902.8-928) $\mathrm{MHz}$ are also available to be used in the biotelemetry applications in addition to the ISM band (2.4 to 2.5) $\mathrm{GHz}$ for the implantable medical devices [6]. Because the human body is electrically lossy in nature with the different tissues of frequency dependent variable permittivity and conductivity in the physical structural form, the implanted antenna has to be operated in the layered lossy media of frequency dispersive material parameters. The electrical field radiated from the implanted antenna and the accompanying magnetic field result the mobile charges in the lossy tissues to experience the Lorentz force with the unwanted heating effect of the tissues. The specific absorption rate (SAR) of the human tissues is restricted to $2 \mathrm{~W} / \mathrm{kg}$ in $10 \mathrm{~g}$ and $1.6 \mathrm{~W} / \mathrm{kg}$ in $1 \mathrm{~g}$ averaged tissue for the general public exposure in IEEE C95.1-2005 [7] and IEEE C95.1-1999 [8] standards, respectively. This design criterion has to be taken into account to determine the communication link margin.

Many research groups have been working in the field of implantable antennas for the realization of wearable wireless medical telemetry systems. The miniaturization methodologies applicable in the implantable antenna design are the use of CPW fed tapered slot radiators on high dielectric substrates, RO3210, with SMP connector type to reduce the antenna overall size for Medical Device Radiocommunication Service (MedRadio) band [9], folded meander PIFA structure with the short circuiting of PIFA geometry on one substrate side to the meander line geome- 
try on the other side for extending the radiating main branches into a spiral shape [10], meandered line dipole radiator with an additional superstrate layer on the substrate for preserving the required biocompatibility [11], [6]. In [12], a conformal miniaturized multilayered spiral antenna is designed for the subcutaneous implantation to have dual-band operation in MedRadio and ISM bands. A compact, probe-fed, broadband implantable antenna based on the patch geometry is also designed for single ISM band biomedical telemetry applications with the circular polarization capability [8], [13]. In [7], an Egret-Beak shaped patch with the shorting pin connected from the radiating patch to the ground plane is designed for the triband operation in MICS, wireless medical telemetry service (WMTS) and ISM bands in wireless medical telemetry applications. The metamaterial inspired geometric structures can also be utilized for the antenna miniaturization to design compact, multifrequency, high gain and efficient implantable antennas as in different RF applications [14-16].

In this paper, a novel, compact, implantable dualband antenna is designed to operate in MICS and ISM bands by taking the lossy human phantom model into account for better RF performance evaluation in further clinical tests. The paper is organized in the following manner. The novel electrically small resonator geometry is explained in Sec. 2 with the design principle. In Sec. 3, the numerical and experimental results of the implantable antenna model are illustrated with the reflection coefficients of the proposed antenna in human body model and free space to figure out the requirement of human body model in the numerical simulations. The surface current distributions are shown to understand the antenna operation principle. The different geometrical parameters of the resonator structure are studied parametrically to point out the design methodology in the optimization process in Sec. 4. In Sec. 5, the antenna radiation patterns and SAR results are illustrated to determine the RF specifications of the bioimplantable transceiver module. The concluding remarks are conducted in Sec. 6.

\section{Bioimplantable Antenna Design}

The proposed compact dual-band bioimplantable antenna model is shown in Fig. 1 along with the geometric parameters. The electrically small resonator model is based on the extended L-shaped direct feeding of anti-spiral resonator structure loaded with the spiral resonator at the end in order to increase the electrical length for the lower band resonant operation. The anti-spiral resonator geometry loaded with the spiral resonator is indicated by the circle in Fig. 1. The L-shaped feeding line is effective for the resonant length in the lower frequency band due to the transmission line loading of anti-spiral resonator for the increased electrical length. The lower substrate side is fully metallized with $18 \mu \mathrm{m}$ thick copper for the ground plane. Because the bioimplantable antennas have to be located in-

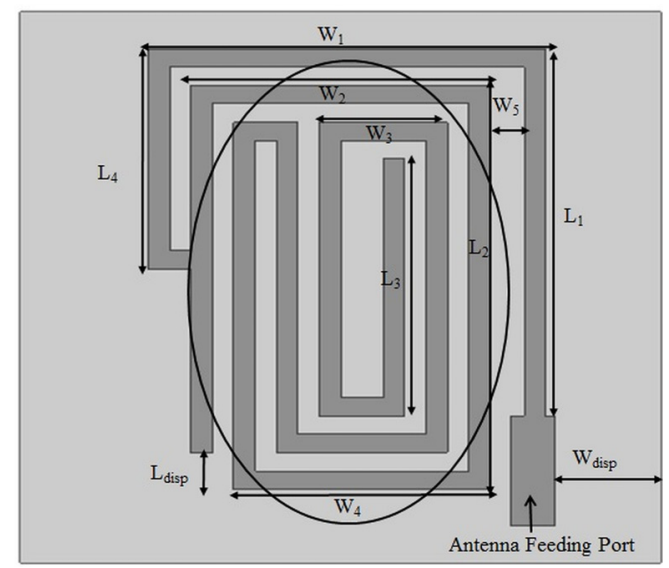

(a)

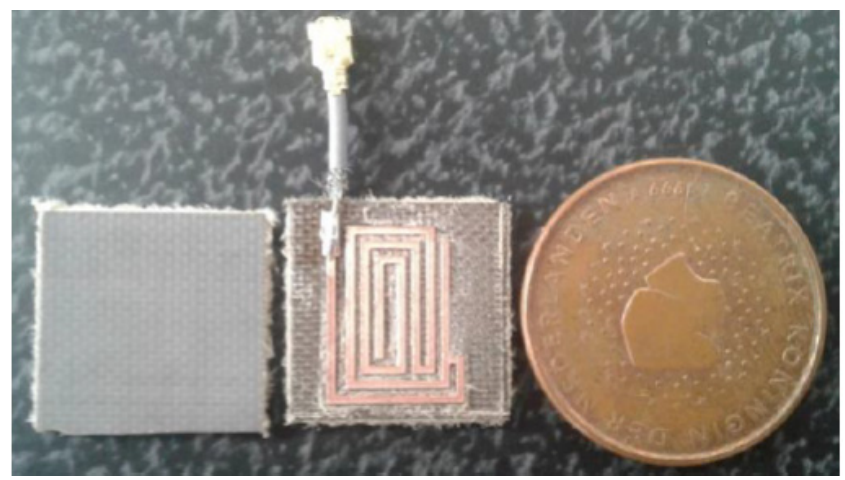

(b)

Fig. 1. (a) Numerical model of dual-band bioimplantable antenna with the geometric parameters of $\mathrm{W}_{1}=11.3 \mathrm{~mm}$, $\mathrm{W}_{2}=9 \mathrm{~mm}, \mathrm{~W}_{3}=5 \mathrm{~mm}, \mathrm{~W}_{4}=8 \mathrm{~mm}, \mathrm{~W}_{5}=0.8 \mathrm{~mm}$, $\mathrm{W}_{\text {sub }}=15 \mathrm{~mm}, \quad \mathrm{~W}_{\text {dis }}=2.5 \mathrm{~mm}, \mathrm{~L}_{1}=9.5 \mathrm{~mm}, \mathrm{~L}_{2}=$ $11 \mathrm{~mm}, \mathrm{~L}_{3}=6.5 \mathrm{~mm}, \mathrm{~L}_{4}=4.5 \mathrm{~mm}, \mathrm{~L}_{5}=5.5 \mathrm{~mm}, \mathrm{~L}_{\text {sub }}=$ $15 \mathrm{~mm}, \mathrm{~L}_{\mathrm{disp}}=4 \mathrm{~mm}$. (b) Fabricated dual-band bioimplantable antenna prototype.

side the conductive human tissue, the lower and upper substrate sides have to be prevented from being short-circuited by the use of dielectric biocompatible superstrates. The substrate and superstrate materials are selected as $0.64 \mathrm{~mm}$ thick Rogers 3210 low loss dielectric material with the relative permittivity of 10.2 and loss tangent of 0.003 . The whole antenna size is $15 \times 15 \times 1.92 \mathrm{~mm}^{3}$, with the surface area of $\lambda_{0} / 20 \times \lambda_{0} / 20$, where $\lambda_{0}$ is the free space wavelength at the lower resonance frequency. The stepped impedance line at the feeding port has $1 \mathrm{~mm}$ width and $3 \mathrm{~mm}$ length to facilitate the soldering of twisted stripped cable U.FL connector. The transmission line width and the distance between any two transmission line sections are $0.5 \mathrm{~mm}$. In the numerical model, the geometric parameters of the electrically small resonator are optimized to have the resonance frequencies in MICS (402-405) MHz and ISM band (2.4-2.5) GHz with the reflection coefficients better than $-25 \mathrm{~dB}$. The higher order modes to be excited at the higher frequencies have negligible effect on the signal propagation through the surrounding lossy body tissues due to the high reflection coefficients. The additional high frequency filtering sections based on the distributed transmission lines can be alternatively added into the resonator 
geometry to filter out the higher order resonance or coupled modes with the expense of larger antenna size and fabrication complexity in the limited space [17], [18].

\section{Numerical and Experimental Results}

The numerical model of compact dual-band bioimplantable antenna has been numerically computed in three layered human body model, composed of skin, fat and muscle layers to optimize the resonator geometric parameters. The 3D fullwave electromagnetic field computations are done in FEM based commercial electromagnetic field solver Ansoft HFSS. The three layered human body model is shown in Fig. 2 along with the finite thicknesses of each layer. The lossy dielectric and density material parameters are listed in Tab. 1. The bioimplantable antenna is located inside the muscle layer with the separation distance of $2 \mathrm{~mm}$ from the lower edge of the fat layer.

In addition to the numerical calculations in the human body model to optimize the reflection coefficients for MICS and ISM operation bands, the numerical and experimental results are obtained to figure out RF performance differences inside the body and outside the body in free space. The reflection coefficients of the fabricated bioimplantable antenna have been measured by Agilent N9912A VNA in free space. The measured and simulated reflection parameters in free space and numerically computed S11 in three layered lossy human model are shown in Fig. 3. As shown in Fig. 3, even though the resonance frequencies can be observed in the numerical computation and experimental measurement results for the proposed bioimplantable antenna in free space, the reflection coefficients are high in the range of $-2 \mathrm{~dB}$, which are not suitable to be used in any kind of wireless radio communication applications. The numerical and experimental results are in

\begin{tabular}{|c|c|c|}
\hline Skin Layer & & $2 \mathrm{~mm}$ \\
\hline \multicolumn{3}{|l|}{ Fat Layer } \\
\hline & & $4 \mathrm{~mm}$ \\
\hline \multirow[t]{2}{*}{ Muscle Layer } & 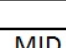 & \\
\hline & MID & $28 \mathrm{~mm}$ \\
\hline
\end{tabular}

Fig. 2. Three layered human body model.

\begin{tabular}{|c|c|c|c|c|}
\hline $\begin{array}{c}\text { Material } \\
\text { Model }\end{array}$ & $\begin{array}{c}\text { Relative } \\
\text { Dielectric } \\
\text { Constant }\end{array}$ & $\boldsymbol{\sigma}[\mathbf{S} / \mathbf{m}]$ & $\begin{array}{c}\text { Density } \\
{\left[\mathbf{k g} / \mathbf{m}^{\mathbf{3}}\right]}\end{array}$ & $\begin{array}{c}\text { Thickness } \\
{[\mathbf{m m}]}\end{array}$ \\
\hline Skin layer & 38.01 & 1.46 & 1100 & 2 \\
\hline Fat layer & 5.28 & 0.1 & 909.4 & 4 \\
\hline Muscle layer & 52.73 & 1.73 & 1040 & 28 \\
\hline
\end{tabular}

Tab. 1. Dielectric and density material parameters of the layered human body model [19]. good agreement for the proposed antenna model in free space. However, because the antenna model optimization has been done by taking the antenna location inside the layered lossy human body model into account in the numerical modelling, S11 is very low as intended in the lower frequency band, which is better than $-10 \mathrm{~dB}$ between $350 \mathrm{MHz}$ and $450 \mathrm{MHz}$ covering the whole MICS band with $25 \mathrm{~dB}$ return loss at $403 \mathrm{MHz}$. For the higher frequency band, $\mathrm{S} 11$ is lower than $-10 \mathrm{~dB}$ in the frequency band of $2.22 \mathrm{GHz}$ and $2.58 \mathrm{GHz}$ with the minimum reflection parameters better than $-40 \mathrm{~dB}$ in two resonance frequencies of $2.34 \mathrm{GHz}$ and $2.45 \mathrm{GHz}$, covering the whole ISM band. The higher order resonance frequencies occurring at the frequencies of $750 \mathrm{MHz}, 980 \mathrm{MHz}, 1.45 \mathrm{MHz}$ and $1.98 \mathrm{MHz}$ have higher reflection coefficients than those at the resonance frequencies of interest in MICS and ISM bands.

The surface current distributions at the resonance frequencies in MICS and ISM bands are shown in Fig. 4. As shown in Fig. 4a, the electrically small resonator has the surface current distribution in the form of quarter wavelength resonance at $403 \mathrm{MHz}$, resulting into the impedance transformation from the open-circuited end of the spiral resonator to the lower impedance end of the antenna foot point location. In Fig. 4b, the higher order resonance can be deduced from the surface current distribution at $2.45 \mathrm{GHz}$

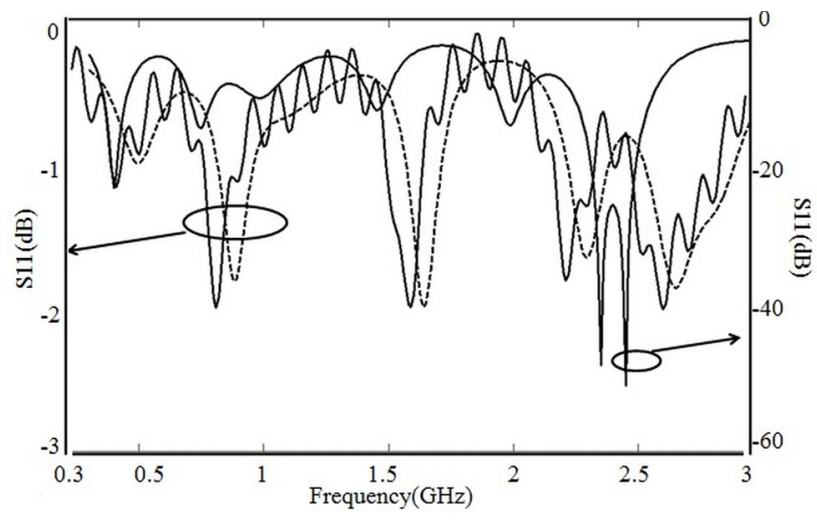

Fig. 3. Simulated (dash) and measured (solid) S11(dB) parameters of dual-band bioimplantable antenna in free space outside the body (left side), simulated reflection coefficient of dual-band bioimplantable antenna inside the body (right side).

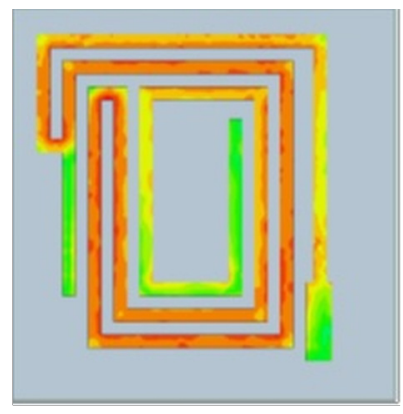

(a)

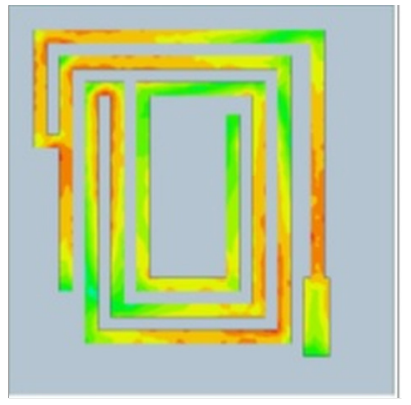

(b)
Fig. 4. Surface current distribution of dual-band bioimplantable antenna at (a) $403 \mathrm{MHz}$ and (b) $2.45 \mathrm{GHz}$. 
due to the excitation of different resonator sections with four effective current nulls in the higher frequency band. To have the antenna resonances at the desired frequencies with the lowest reflection coefficients is not the only important design criterion. Permissible signal reception to and transmission from the bioimplantable sensor module in the biotelemetry systems are also important RF system parameters to be investigated with the possibly high radiation efficiency.

\section{Numerical Parametric Studies}

In the bioimplantable antenna design for the biotelemetry applications, it is very important to reduce the antenna size in an effective manner in order for the antenna to be placed inside the encapsulated bioimplanted transceiver $\mathrm{RF}$ electronics module. It is therefore crucial to compute the antenna performance parameters inside the conductive human body with the lossy dielectric material properties. Therefore, the numerical parametric studies in the proposed bioimplantable antenna model are done for the optimization of RF antenna performance. As a starting point to determine the resonant antenna length at the lower frequency band, the total line length from the feeding line to the end of the spiral resonator is taken to be equal to $\lambda_{\mathrm{g}} / 4$, where $\lambda_{\mathrm{g}}$ is the guided wavelength in the substrate $[17,20]$ as it can be deduced from the surface current distribution in Fig. 4a. The total length of extended L-shaped feeding line, anti-spiral resonator and spiral resonator sections are parametrically changed to satisfy the input impedance at MICS and ISM bands for the final prototype. Therefore, three geometric parameters, $\mathrm{L}_{\mathrm{disp}}, \mathrm{L}_{4}$ and $\mathrm{L}_{3}$ are numerically studied to figure out the effect of antenna geometry on the resonance frequency in this section.

\subsection{Displacement Length between Antispiral Resonator and Substrate Edge}

The first geometric parameter to be numerically studied is the displacement distance between the extended length of anti-spiral resonator and lower substrate edge

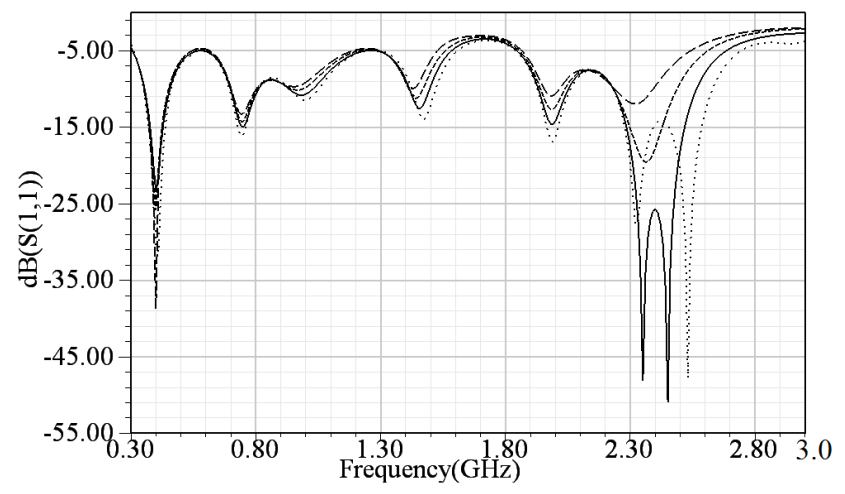

Fig. 5. S11 parameter of the dual-band bioimplantable antenna for different $\mathrm{L}_{\text {disp }}$ in three layered human body model (dot: $5 \mathrm{~mm}$, solid: $4 \mathrm{~mm}$; dash: $3 \mathrm{~mm}$; long dash: $2 \mathrm{~mm})$. indicated in Fig. 1. The reflection coefficient (S11) is numerically computed for different $\mathrm{L}_{\text {disp }}$ values to figure out the effect of anti-spiral resonator length on S11 as shown in Fig. 5. As shown in Fig. 5, the resonance frequency in the higher frequency band is increasing for the reduced length of anti-spiral resonator with the minor frequency shift in the lower frequency band. Due to the effect of resonator coupling for the larger displacements from the substrate edge, the single resonance frequency of the loosely coupled two modes is split into two resonance frequencies of two modes at the higher frequency.

\subsection{Extended L-shaped Transmission Line and Antispiral Resonator Connection Position $\left(\mathbf{L}_{4}\right)$}

The second geometric parameter to be parametrically analyzed is the position where the extended L-shaped feeding line is directly connected to the spiral resonator loaded anti-spiral resonator, which is indicated as length $\mathrm{L}_{4}$. The reflection coefficient S11 is computed for different $\mathrm{L}_{4}$ values to determine how the location of galvanically connected L-shaped feeding line to the anti-spiral resonator affects the antenna resonance frequency as shown in Fig. 6. As shown in Fig. 6, the resonance frequency in the higher frequency band is emerging predominantly for larger $\mathrm{L}_{4}$ due to the increment in the resonant length and resulting input impedance matching. Increasing resonator length enhances the near field reactive coupling to the surrounding lossy dielectric body tissues with the result of increased bandwidth and splitting of the mode at the resonance frequency into two different nondegenerate modes observed in highly coupled resonators [17]. The frequency shift is minor for various $\mathrm{L}_{4}$ values in the lower frequency band due to the small change in the resonator length with respect to the resonant length at the lower resonance frequency.

\subsection{Inner Spiral Resonator Length of Spiral Resonator Loaded Anti-Spiral Resonator $\left(\mathbf{L}_{3}\right)$}

The third geometric parameter to be investigated is the length of the inner spiral resonator, which is in the form of open-circuited spiral transmission line termination of anti-spiral resonator, indicated as length $\mathrm{L}_{3}$. As shown in Fig. 7, the resonance frequencies of the lower and higher frequency bands are decreasing for larger $L_{3}$ due to the increment in the resonant length of spiral resonator. Larger spiral resonator length improves the near field coupling of different sections of anti-spiral resonator and L-shaped extended transmission line sections in a form of increased bandwidth with better return loss at the higher frequency band. The similar frequency dependency of S11 has been observed in the geometric parametric studies of $\mathrm{L}_{4}$ and $\mathrm{L}_{\text {disp. }}$. The fundamental reason of increased bandwidth is not only the effect of capacitive and inductive coupling of the main radiating resonator sections but also the near field 


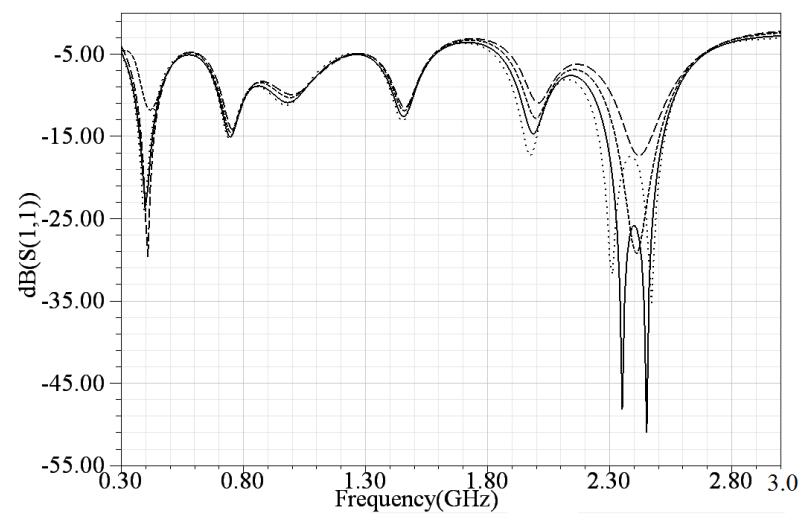

Fig. 6. S11 parameter of the dual-band bioimplantable antenna for different $\mathrm{L}_{4}$ in three layered human body model (dot: $5 \mathrm{~mm}$, solid: $4.5 \mathrm{~mm}$; dash: $4 \mathrm{~mm}$; long dash: $3.5 \mathrm{~mm}$ ).

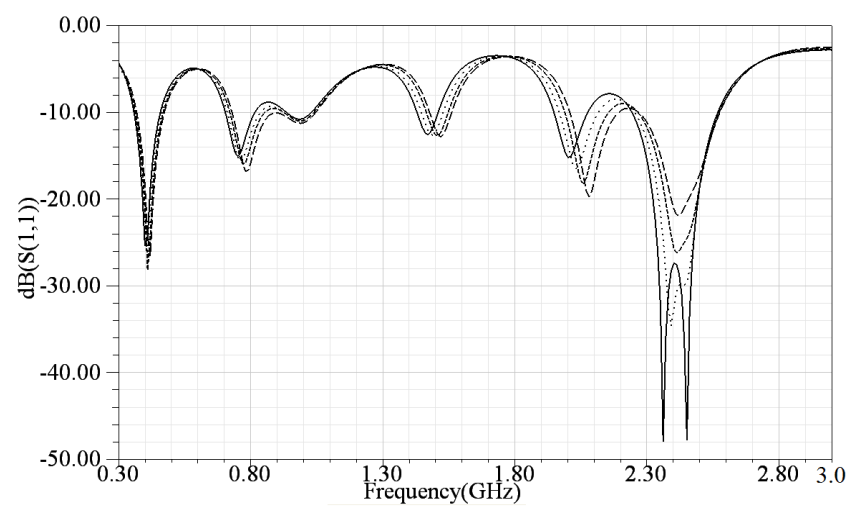

Fig. 7. S11 parameter of the dual-band bioimplantable antenna for different $\mathrm{L}_{3}$ in three layered human body model (solid: $6 \mathrm{~mm}$, dot: $5 \mathrm{~mm}$, dash: $4 \mathrm{~mm}$, long dash: $3 \mathrm{~mm})$.

coupling of different transmission line sections to the surrounding lossy human tissues of lower Q. The electromagnetic coupling decreases the loaded $\mathrm{Q}$, which deteriorates the radiation efficiency and gain at the higher frequency band.

\section{Antenna Radiation Parameters}

In addition to the determination of the optimum geometric parameters for the return loss, the RF radiation parameters of the proposed antenna have to be investigated for the reliable operation of the implantable transceiver module in the wireless communication link. The $3 \mathrm{D}$ radiation patterns of the proposed implantable antenna are shown in Fig. 8 at the resonance frequencies of the lower and higher frequency bands. The gain values at $403 \mathrm{MHz}$ and $2.45 \mathrm{GHz}$ are $-12.25 \mathrm{dBi}$ and $-12.4 \mathrm{dBi}$ with the directivity values of $1.076 \mathrm{dBi}$ and $4.16 \mathrm{dBi}$ in MICS and ISM bands, respectively. Because of the propagation of the excited electromagnetic field inside the lossy medium, the antenna gain values at the operation frequencies are low with the maximum radiation along the direction perpendicular to the implantable antenna surface. The ground plane is electrically small in the size of $\lambda_{0} / 20 \times \lambda_{0} / 20$ of the free space wavelength at the lower resonance frequency, which results into the wave propagation to be more effective to the opposite direction in comparison to the wave radiation at the higher frequency band as in Fig. 8b. The phase center of the radiation pattern is assumed to be the central location of the proposed implantable antenna. However, due to the lossy nature of the human tissues, the determination of the phase center for the radiation pattern is uncertain, which is resulting from the various tissue locations having different contributions to the total radiated fields. On the other hand, to prevent the patients from the electromagnetic damage resulting from the implantable antenna, SAR restrictions for the public exposure have to be satisfied. The implantable antenna has to have the SAR limit specification IEEE C95.1-1999 of $1.6 \mathrm{~W} / \mathrm{kg}$ in $1 \mathrm{~g}$ averaged tissue. Therefore, SAR distributions on the superstrate surfaces are numerically calculated and shown in Fig. 9 for the total delivered input power of $1 \mathrm{~W}$ to the antenna. As shown in Fig. 9, the maximum SAR values are observed at the central location of the radiating element with decreasing heat transformed dissipated energy far from the antenna center. The delivered power level has to be decreased to $1.6 \mathrm{~mW}$ to meet the SAR specification in both operation frequency bands. These are important design considerations while designing the whole implanted transceiver module with the careful attention given to SAR limitations.

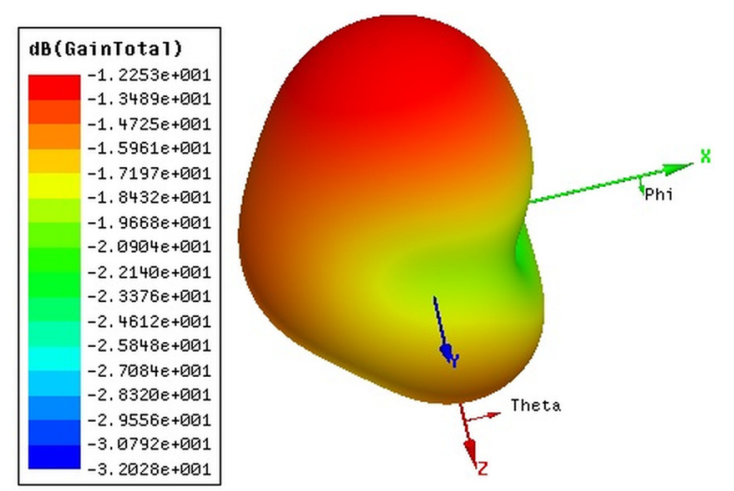

(a)
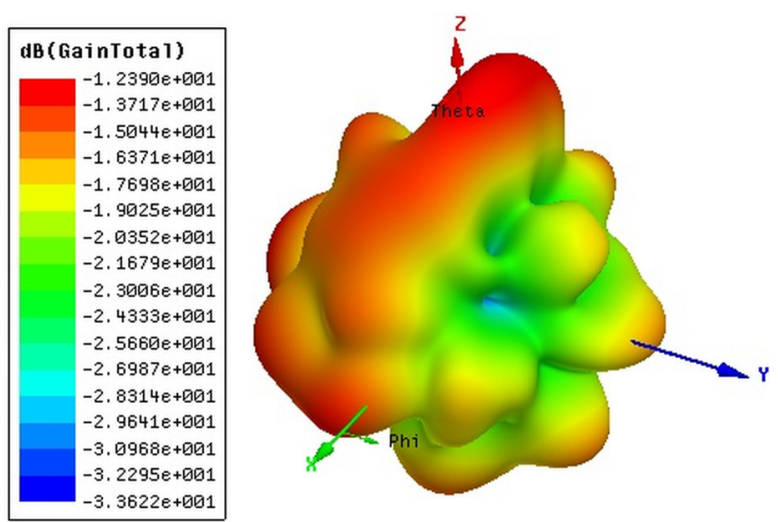

(b)

Fig. 8. 3D radiation patterns of dual-band bioimplantable antenna at (a) $403 \mathrm{MHz}$ and (b) $2.45 \mathrm{GHz}$. 


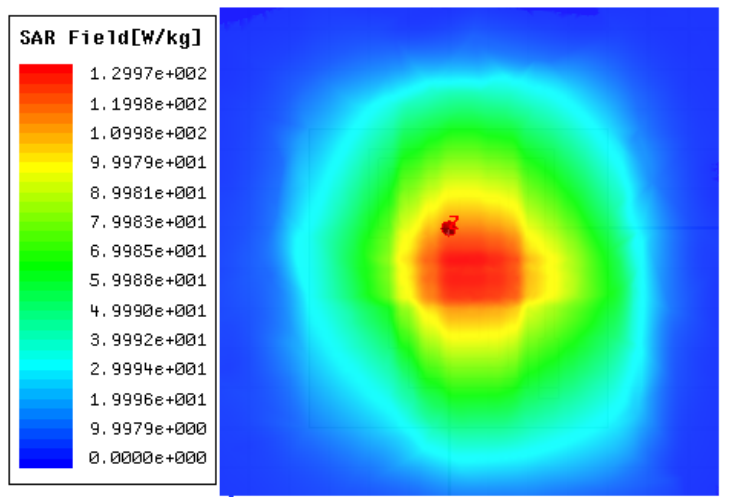

(a)

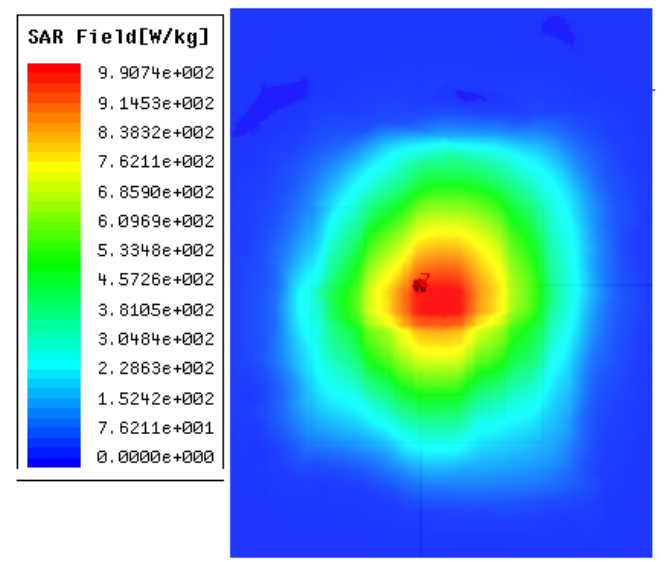

(b)

Fig. 9. Average SAR distribution of dual-band bioimplantable antenna at (a) $403 \mathrm{MHz}$ and (b) $2.45 \mathrm{GHz}$ for $1 \mathrm{~W}$ delivered antenna input power.

\section{Conclusion}

In this paper, a compact bioimplantable antenna with novel electrically small geometry is designed for the dualband operation in MICS and ISM band wireless biotelemetry applications. The numerical calculation and experimental measurement results are illustrated.

The importance of three layered lossy human tissue model is pointed out with the simulation and experimental results in free space. The proposed antenna has the impedance bandwidth of $100 \mathrm{MHz}$ at $403 \mathrm{MHz}$ and $360 \mathrm{MHz}$ at $2.45 \mathrm{GHz}$, covering the whole MICS and ISM band. The design and operation principles of the novel resonator geometry are explained with the numerically computed surface current distributions. The proposed antenna has the total volume of $432 \mathrm{~mm}^{3}$, which is very compact. The effects of the resonator geometrical parameters on the return loss are figured out. The computed SAR values at the resonance frequencies indicate the input power delivered to the antenna has to be $1.6 \mathrm{~mW}$. The radiation parameters and $3 \mathrm{D}$ radiation patterns indicate the potential use of the proposed implantable antenna having the permissible gains in the range of $-12.4 \mathrm{dBi}$ at maximum in dual-band wireless biotelemetry applications.

\section{References}

[1] SKRIVERVIK, A. K. Implantable antennas: The challenge of efficiency. In The 7th European Conference on IEEE Antennas and Propagation (EuCAP). Gothenburg (Sweden), 2013, p. 3627 to 3631 .

[2] ASILI, M., GREEN, R., SERAN, S., et al. A small implantable antenna for MedRadio and ISM bands. IEEE Antennas and Wireless Propagation Letters, 2012, vol. 11, p. 1683-1685. DOI: 10.1109/LAWP.2013.2241723

[3] International Telecommunications Union-Radio Communications, (ITUR), Radio Regulations, SA.1346, ITU, Geneva (Switzerland).

[4] Medical Implant Communication Service (MICS) Federal Register, Rules Regulations, 64, 240, December 1999, p. 69926 to 69934.

[5] European Radiocommunications Commission Recommendation 70-03 Relating to the Use of Short Range Devices. In European Conference of Postal and Telecommunications Administration, CEPT/ERC 70-03, Annex 12, 1997.

[6] FERDOUS, N., NAINEE, N. T., HOQUE, R. Design and performance of miniaturized meandered patch antenna for implantable biomedical applications. In The 2nd IEEE International Conference on Electrical Engineering and Information Communication Technology (ICEEICT). Dhaka (Bangladesh), 2015. DOI: 10.1109/ICEEICT.2015.7307370

[7] SHAKIB, M., MOGHAVVEMI, M., MAHADI, W., et al. Design of a tri-band implantable antenna for wireless telemetry applications. In IEEE MTT-S International Microwave Workshop Series on RF and Wireless Technologies for Biomedical and Healthcare Applications (IMWS-Bio). London (UK), 2014. DOI: 10.1109/IMWS-BIO.2014.7032451

[8] LI, H., GUO, Y.-X., XIAO, S.-Q. Broadband circularly polarised implantable antenna for biomedical applications. Electronics Letters, 2016, vol. 52, no. 7, p. 504-506. DOI: $10.1049 / \mathrm{el} .2015 .4445$

[9] SHARMA, M., PARINI, C. G. A miniature wideband antenna for wearable systems. In IEEE Antennas and Propagation Conference $(L A P C)$. Loughborough (UK), 2013, p. 619-623. DOI: 10.1109/LAPC.2013.6711975

[10] HUANG, C.-Y., TSAI, C. -L., YANG, C.-L. Compact folded meander PIFA antennas in MedRadio bands. In Progress In Electromagnetics Research Symposium Proceedings. Tapei, 2013, p. 756-759.

[11] UNG, J., KARACOLAK, T. A dual-band meandered dipole antenna for medical telemetry applications. Progress In Electromagnetics Research C, 2016, vol. 63, p. 85-94. DOI: 10.2528/PIERC16012603

[12] MERLI, F., BOLOMEY, L., ZÜRCHER, J.-F., et al. Design, realization and measurements of a miniature antenna for implantable wireless communication systems. IEEE Transactions on Antennas and Propagation, 2011, vol. 59, no. 10, p. 3544-3555. DOI: 10.1109/TAP.2011.2163763

[13] LIU, C., GUO, Y.-X., JEGADEESAN R., et al. In vivo testing of circularly polarized implantable antennas in rats. IEEE Antennas and Wireless Propagation Letters, 2015, vol. 14, p. 783-786. DOI: 10.1109/LAWP.2014.2382559

[14] PALANDOKEN, M. Artificial Materials Based Microstrip Antenna Design. Chapter 3 in Microstrip Antennas. Ed. Nasimuddin, N. INTECH Open Access Publisher, 2011, ISBN: 978-953-307-247-0

[15] PALANDOKEN, M., HENKE, H. Fractal negative-epsilon metamaterial. In IEEE International Workshop on Antenna 
Technology (iWAT). Lisbon (Portugal), 2010. DOI: 10.1109/IWAT.2010.5464790

[16] PALANDOKEN, M., HENKE, H. Fractal spiral resonator as magnetic metamaterial. In IEEE Applied Electromagnetics Conference (AEMC). Kolkata (India), 2009. DOI: 10.1109/AEMC.2009.5430645

[17] POZAR, D. M. Microwave Engineering. $4^{\text {th }}$ ed., John Wiley \& Sons, 2011. ISBN-10: 0470631554

[18] PALANDOKEN, M. Metamaterial-Based Compact Filter Design. Chapter 19 in Metamaterial. Ed. Xun-Ya Jiang. INTECH Open Access Publisher, 2012. ISBN: 978-953-51-0591-6

[19] LOKTONGBAM, P., LAISHRAM, R. Performance and design analysis of an implantable antenna for biotelemetry. International Journal of Scientific and Research Publications, 2017, vol. 7, no. 6, p. 105-114. ISSN: 2250-3153
[20] BALANIS, C. A. Antenna Theory: Analysis and Design. $3^{\text {rd }}$ ed., Wiley-Interscience, 2005. ISBN-13:978-0471667827
About the Author ...
Merih PALANDÖKEN was born in Kahramanmaras, Turkey. He received his M.Sc. degree in the field of Mi- crosystem and Microelectronics Engineering from Tech- nische Universitaet Hamburg, Germany in 2005 and Ph.D. degree in the field of Metamaterial Based Antennas and Microwave Filters from the Dept. of Theoretical Electrical Engineering at Technische Universitaet Berlin, Germany in 2012. His research interests include bioimplantable antenna design, RF energy harvesting system design, electromag- netic field theory and electrically small antenna design. 